\title{
Real-Time Three-Dimensional Display Based on the Partial Pixel Architecture
}

Gregory P. Nordin

nordin@byu.edu

M. W. Jones

S. T. Kowel

J. H. Kulick

R. G. Lindquist

Follow this and additional works at: https://scholarsarchive.byu.edu/facpub

Part of the Electrical and Computer Engineering Commons

\section{Original Publication Citation}

M. W. Jones, G. P. Nordin, J. H. Kulick, R. G. Lindquist, and S. T. Kowel,"Real-Time ThreeDimensional Display Based on the Partial Pixel Architecture" Opt. Lett. 2(12), pp. 1418-142 (1995)

\section{BYU ScholarsArchive Citation}

Nordin, Gregory P.; Jones, M. W.; Kowel, S. T.; Kulick, J. H.; and Lindquist, R. G., "Real-Time ThreeDimensional Display Based on the Partial Pixel Architecture" (1995). Faculty Publications. 1157. https://scholarsarchive.byu.edu/facpub/1157 accepted for inclusion in Faculty Publications by an authorized administrator of BYU ScholarsArchive. For more information, please contact ellen_amatangelo@byu.edu. 


\title{
Real-time three-dimensional display based on the partial pixel architecture
}

\author{
M. W. Jones, G. P. Nordin, J. H. Kulick, R. G. Lindquist, and S. T. Kowel \\ Department of Electrical and Computer Engineering, The University of Alabama in Huntsville, Huntsville, Alabama 35899
}

Received March 9, 1995

\begin{abstract}
We previously reported several static three-dimensional (3-D) display implementations of the partial pixel architecture [J. Opt. Soc. Am. A 12, 73 (1995)]. We report herein our first real-time 3-D display based on this architecture. The display is functionally equivalent to a real-time holographic stereogram. It is autostereoscopic and provides horizontal motion parallax. The display device is composed of a diffractive optical element (fabricated with standard photolithographic techniques) and a separate conventional liquidcrystal display. The display has been used to play back a precomputed animated 3-D scene at video frame rates using a standard VGA video output.
\end{abstract}

In recent years there has been renewed interest in realtime three-dimensional (3-D) display systems. ${ }^{1-4}$ We previously reported the conceptual development of a 3 -D display architecture (the partial pixel architecture $^{5}$ ) that is equivalent to a real-time holographic stereogram. ${ }^{6} \quad$ A key advantage of this architecture is that the computational load required for driving the display is reduced to the calculation of sets of twodimensional (2-D) images by use of standard graphics algorithms instead of calculating (and generating) holographic fringes from first principles. Furthermore the architecture utilizes space-bandwidth products that are compatible with current VLSI technology. We previously demonstrated the 3-D image-forming properties of this architecture with several devices that displayed static 3 -D scenes. ${ }^{7,8}$ In this Letter we report our first real-time 3-D display based on the partial pixel architecture. ${ }^{9}$

The basic geometry of the partial pixel architecture is shown in Fig. 1. It consists of a pixelated display and a well-defined viewing region located at a distance $d_{V}$ from the display. The viewing region is divided into a series of virtual viewing slits, each of which is approximately one pupil diameter wide. A unique 2-D image is visible from each virtual viewing slit. When an appropriate pair of images (i.e., a stereoimage pair) is simultaneously seen by the left and right eyes the scene appears to be three dimensional. Horizontal motion parallax is provided by displaying multiple stereo-image pairs.

For a pixelated device to display multiple 2-D images simultaneously, each pixel must be able to exhibit a different appearance, or brightness, when viewed from different virtual viewing slits. ${ }^{10}$ We accomplish this by dividing the pixel into spatially distinct regions, referred to as partial pixels. As described in Ref. 5, each partial pixel is responsible for the overall pixel's appearance from a particular virtual viewing slit. Diffraction gratings are placed in each partial pixel to direct the incident light into the appropriate virtual viewing slit. Modulation of the light passing through a partial pixel is accomplished by either varying the diffraction efficiency of the gratings ${ }^{8}$ or using a separate spatial light modulator element. ${ }^{5}$ Diffraction from the aperture of the partial pixel is used to define the physical extent of the virtual viewing slit. ${ }^{10}$

Our prototype real-time 3-D display is shown schematically in Fig. 2. The display consists of a diffractive optical element (DOE) and a separate conventional liquid-crystal display (LCD) from which the polarizers have been removed. As shown in Fig. 3, the LCD is mounted to the DOE with a special fixture that allows the LCD to be aligned to the DOE to within a few micrometers. The LCD/DOE assembly is placed between crossed polarizers. The LCD is a miniature VGA-compatible monochrome active matrix twisted nematic LCD manufactured by Kopin Corporation. This LCD was chosen primarily for its ability to independently address individual LCD pixels. The LCD pixels have a clear aperture of $40 \mu \mathrm{m} \times 40 \mu \mathrm{m}$ and a pitch of $55 \mu \mathrm{m}$ in both directions. The DOE is an array of amplitude diffraction gratings, with one grating for each partial pixel in the display. The gratings are etched in chrome on a quartz integrated circuit mask and have a $50 \%$ duty cycle. The minimum feature size is approximately $1 \mu \mathrm{m}$. The gratings each have an aperture of $45 \mu \mathrm{m} \times 25 \mu \mathrm{m}$, which results in an effective partial pixel aperture (defined by the overlap of the grating aperture and the LCD pixel clear aperture) of $25 \mu \mathrm{m} \times 40 \mu \mathrm{m}$. This is illustrated in Fig. 3 .

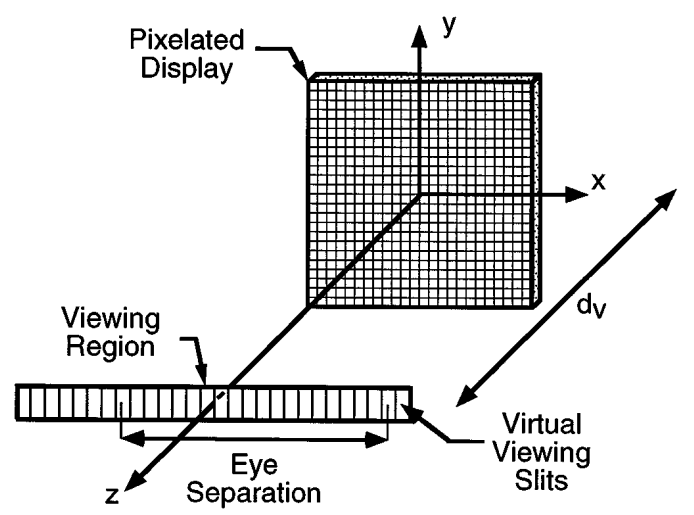

Fig. 1. Geometry of the partial pixel architecture (after Ref. 5). 


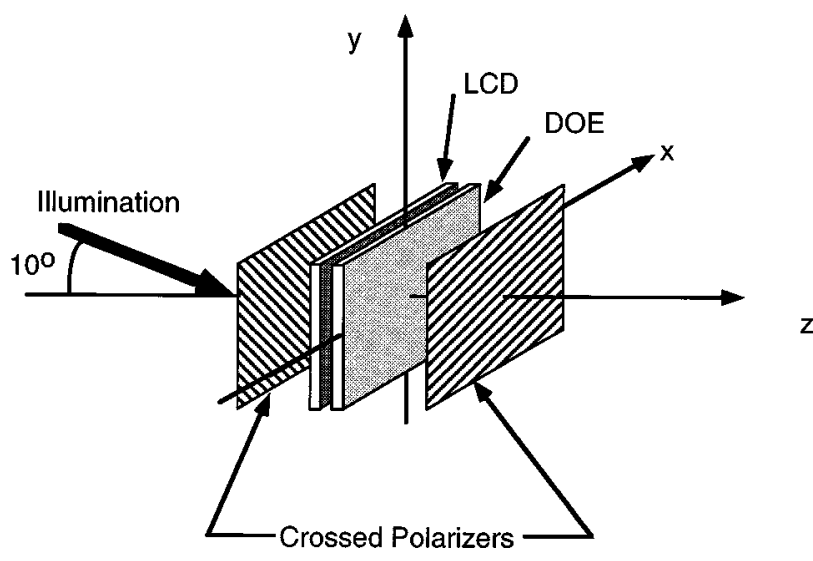

(a)

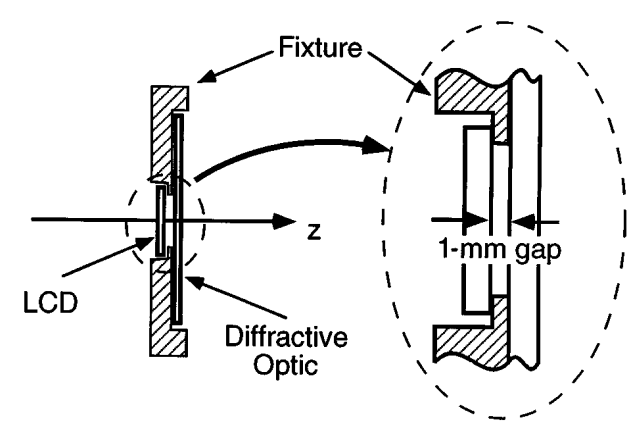

(b)

Fig. 2. (a) Device configuration and readout geometry, (b) close-up of LCD and DOE mounting.

As shown in Fig. 4, the display is designed to be viewed from $30 \mathrm{~cm}$ when it is illuminated by an incoherent quasi-monochromatic (10-nm-bandwidth) collimated beam centered at $630 \mathrm{~nm}$. The viewing region consists of 16 virtual viewing slits positioned such that 8 distinct stereo-image pairs are displayed. Each virtual viewing slit is approximately $6 \mathrm{~mm}$ wide by $10 \mathrm{~mm}$ tall. The maximum angular viewing range of the display, which is determined by the minimum grating pitch in the DOE, is $\pm 10.8^{\circ}$. The pixels, which are composed of a $4 \times 4$ array of partial pixels, are $220 \mu \mathrm{m} \times 220 \mu \mathrm{m}$. The display consists of a $135 \times 90$ array of pixels, giving a total of 194,400 partial pixels and corresponding diffraction gratings. The display area is $29.7 \mathrm{~mm} \times 19.8 \mathrm{~mm}$.

The device was illuminated as shown in Fig. with one of two separate sources (either a mercury-vapor arc lamp or a MagLite flashlight), and a precomputed animated 3-D sequence was displayed. For both illumination sources the animated scene was bright, clearly visible, and readily fused into a 3-D scene. As with our previous static devices, one-dimensional motion parallax was perceived as the observer horizontally traversed the viewing region. A typical stereo-image pair is shown in Fig. 5. The different 2-D perspectives of the 3-D object are clearly visible.

Secondary images are also visible in these images. An example is the apparent blurring of the upper left cylinder in Fig. 5(a). One source of the secondary images is cross talk in the LCD. When a particular
LCD pixel is addressed, LCD pixels on either side are also partially turned on. This causes the corresponding pixel to appear to be on in virtual viewing slits, from which it should appear to be off. Thus dimmer copies of a 2-D image intended for one virtual viewing slit appear in other virtual viewing slits. The intensity of the secondary images was minimized, but not completely eliminated, by adjustment of the brightness and contrast settings of the LCD display.

The presence of such residual secondary images can be attributed to divergence of the readout illumination. In this case, light leaving an LCD pixel spreads out and is incident upon several partial pixels. As with the LCD cross talk, multiple partial pixels appear to be on when a single partial pixel is addressed. Use of the MagLite as an illumination source resulted in more pronounced secondary images because of a lower degree of readout beam collimation than when the mercury-vapor arc lamp was used. We can minimize the intensity of the secondary images resulting from beam divergence by decreasing the spacing between the LCD and DOE (which is approximately $1 \mathrm{~mm}$ in our current device) and by improving the collimation of the source.

In addition to the qualitative evaluation above, we measured the contrast ratio and overall efficiency of

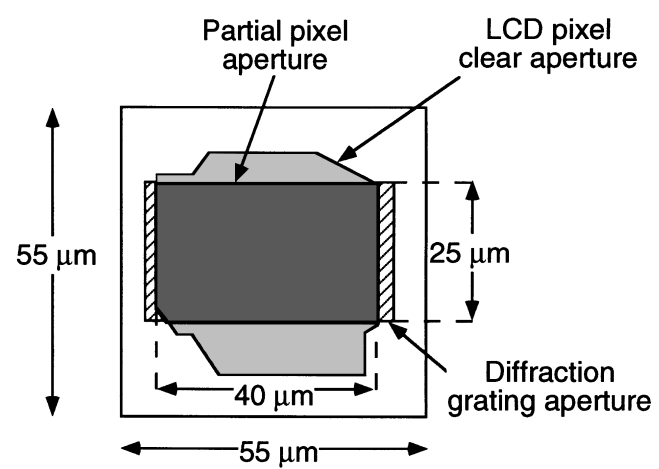

Fig. 3. Geometry of the partial pixel. The darkly shaded region represents the effective partial pixel aperture.

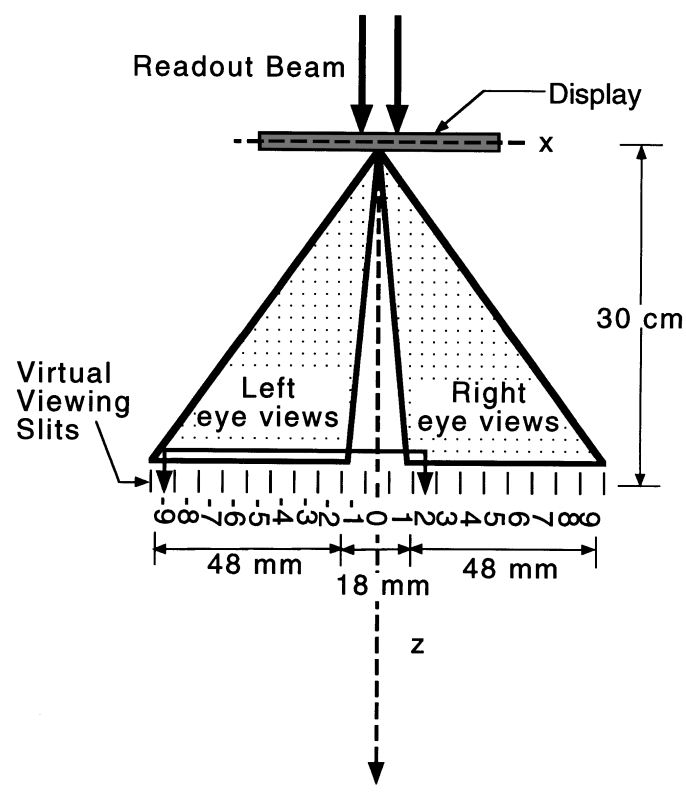

Fig. 4. Geometry of the viewing region. 


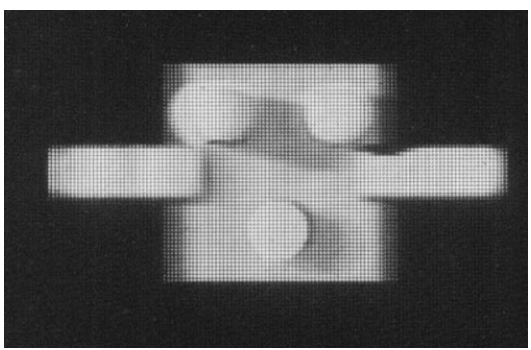

(a)

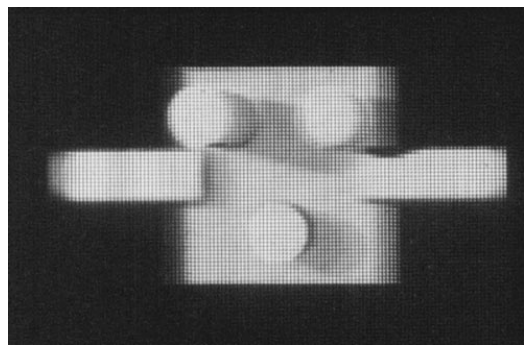

(b)

Fig. 5. (a) Typical left-eye image, (b) typical right-eye image.

our display. The maximum contrast ratio (defined as the ratio of the power transmitted by a pixel when it is fully on to the power transmitted when the pixel is completely off) is approximately 100 . The overall optical efficiency of the device is dependent on the efficiency of both the LCD and the DOE as well as on the geometric fill factor of the partial pixel (see Fig. 4). We define the efficiency, $E$, of our display as the ratio of the optical power directed into the pupil of an observer's eye by a partial pixel to the total optical power incident on the partial pixel. This can be expressed as

$$
E=\eta_{p} E_{\mathrm{LCD}} F,
$$

in which $\eta_{p}$ is the usable diffraction efficiency of the grating (defined as the percentage of the +1 order that actually enters the pupil of an observer's eye $\left.{ }^{8}\right), E_{\mathrm{LCD}}$ is the efficiency of the LCD, and $F$ is the partial pixel geometric fill factor. The efficiency of the display was $0.07 \%$, with $E_{\mathrm{LCD}}=10 \%, \eta_{p}=2.1 \%$, and $F=33 \%$. This efficiency is sufficient that a collimated beam with an intensity of approximately $8 \mathrm{~mW} / \mathrm{m}^{2}$ will produce a display brightness of 20 lumens $/ \mathrm{m}^{2} \mathrm{~s}$ (which is typical of color computer monitors).

In summary, we have demonstrated a real-time 3-D display based on the partial pixel architecture. The display exhibited excellent contrast and brightness with both arc lamp and flashlight illumination. Some degradation in image quality was observed as a result of secondary images. We are currently implementing a full-color real-time version of this display. Future research efforts include examining the use of silicon substrates $^{8}$ such that the computational and drive electronics can be buried below a reflective display layer. ${ }^{5}$ One may realize large displays by tessellating multiple silicon dies.
This project was supported by the Advanced Research Projects Agency and the U.S. Army Missile Command in Huntsville, Alabama. We thank Aness Ahmad and Deborah Bailey for their assistance in design of the alignment fixture and Ahmed Siddique for his assistance in software development.

\section{References}

1. P. St. Hilaire, S. A. Benton, M. Lucente, M. L. Jepson, J. Kollin, H. Yoshikawa, and J. Underkoffler, Proc. Soc. Photo-Opt. Instrum. Eng. 1212, 174 (1990).

2. N. Hashimoto and S. Morokawa, J. Electr. Imag. 2, 93 (1993).

3. L. Onural, G. Bozdagi, and A. Atalar, Opt. Eng. 33, 835 (1994).

4. T. Toda, S. Takahashi, and F. Iwate, "3D video system using a grating image," Proc. Soc. Photo-Opt. Instrum. Eng. 2406, 191 (1995).

5. J. H. Kulick, G. P. Nordin, A. Parker, S. T. Kowel, R. G. Lindquist, M. Jones, and P. Nasiatka, J. Opt. Soc. Am. A 12, 73 (1995).

6. S. A. Benton, Proc. Soc. Photo-Opt. Instrum. Eng. 367, 15 (1982).

7. G. P. Nordin, J. H. Kulick, M. Jones, P. Nasiatka, R. G. Lindquist, and S. T. Kowel, Opt. Lett. 19, 901 (1994).

8. G. P. Nordin, J. H. Kulick, R. G. Lindquist, P. J. Nasiatka, M. W. Jones, M. Friends, and S. T. Kowel, "Liquid-crystal-on-silicon implementation of the partial pixel three-dimensional display architecture," Appl. Opt. (to be published).

9. M. W. Jones, G. P. Nordin, J. H. Kulick, G. Lindquist, and S. T. Kowel, "A liquid crystal display based implementation of a real-time ICVision holographic stereogram display, Proc. Soc. Photo-Opt. Instrum. Eng. 2406, 154 (1995).

10. J. Kulick, S. Kowel, G. Nordin, A. Parker, R. Lindquist, P. Nasiatka, and M. Jones, Proc. Soc. Photo-Opt. Instrum. Eng. 2176, 2 (1994). 